Published in final edited form as:

ACS Infect Dis. 2016 July 08; 2(7): 500-508. doi:10.1021/acsinfecdis.6b00052.

\title{
Diphenylether-modified 1,2-diamines with improved drug properties for development against Mycobacterium tuberculosis
}

\author{
Marie H. Foss ${ }^{1}$, Sovitj Pou², Patrick M. Davidson ${ }^{1}$, Jennifer L. Dunaj ${ }^{1}$, Rolf W. Winter ${ }^{2}$, \\ Sovijja Pou ${ }^{2}$, Meredith H. Licon ${ }^{1}$, Julia K. Doh ${ }^{1}$, Yuexin Li $^{2}$, Jane X. Kelly², Rozalia A. \\ Dodean $^{2}$, Dennis R. Koop ${ }^{3}$, Michael K. Riscoe ${ }^{1,2}$, Georgiana E. Purdy ${ }^{1}$ \\ 1.Department of Molecular Microbiology \& Immunology, Oregon Health \& Science University, 3181 \\ SW Sam Jackson Park Road, Portland, OR, 97239. \\ 2.Portland VA Medical Center, 3710 SW US Veterans Hospital Road, Portland, OR, 97239. \\ 3.Department of Physiology and Pharmacology, Oregon Health \& Science University, 3181 SW \\ Sam Jackson Park Road, Portland, OR, 97239.
}

\begin{abstract}
New treatments for tuberculosis infection are critical to combat the emergence of multi-drug and extensively-drug resistant Mycobacterium tuberculosis (Mtb). We report the characterization of a diphenylether modified adamantyl 1,2-diamine that we refer to as TBL-140, which has a minimal inhibitory concentration ( $\mathrm{MIC}_{99}$ ) of $1.2 \mu \mathrm{g} / \mathrm{mL}$. TBL-140 is effective against drug-resistant $\mathrm{Mtb}$ and non-replicating bacteria. In addition, TBL-140 eliminates expansion of Mtb in cell culture infection assays at its MIC. To define the mechanism of action of this compound, we performed a spontaneous mutant screen and biochemical assays. We determined that TBL-140 treatment impacts the proton motive force (PMF) by perturbing the transmembrane potential $(\Delta \Psi)$, consistent with a target in the electron transport chain (ETC). As a result, treated bacteria have reduced intracellular ATP levels. We show that TBL-140 exhibits greater metabolic stability than SQ109, a structurally similar compound in clinical trials for treatment of MDR-TB infections. Combined, these results suggest that TBL-140 should be investigated further to assess its potential as an improved therapeutic lead against Mtb.
\end{abstract}

\section{Graphical Abstract}

\footnotetext{
Corresponding Author (G.E.P.) Phone: (503) 346-0767 purdyg@ ohsu.edu.

Author contributions

G.E.P and M.K.R designed the research; S.P., R.W.W. and S.P. synthesized compounds; M.H.F, P.M.D, J.L.D., M.H.L, J.K.D, Y.L, J.X.K, R.A.D and G.E.P performed experiments; D.R.K. performed metabolic stability analysis; M.H.F., G.E.P. and M.K.R analyzed data; M.H.F. and G.E.P wrote the paper with contributions from other authors.

The authors declare no competing financial interest.

Supporting Information

MICs of bis-adamantyl derivatives and $\mathbf{8}$ against Mtb, synthesis schemes for compounds, full synthesis and characterization for all compounds, Ramachandran plot of MmpL3 structure model, effect of SQ109 on $\Delta \Psi$ and $\Delta \mathrm{pH}$ in M. bovis BCG. This information is available free of charge via the Internet at http://pubs.acs.org/.
} 


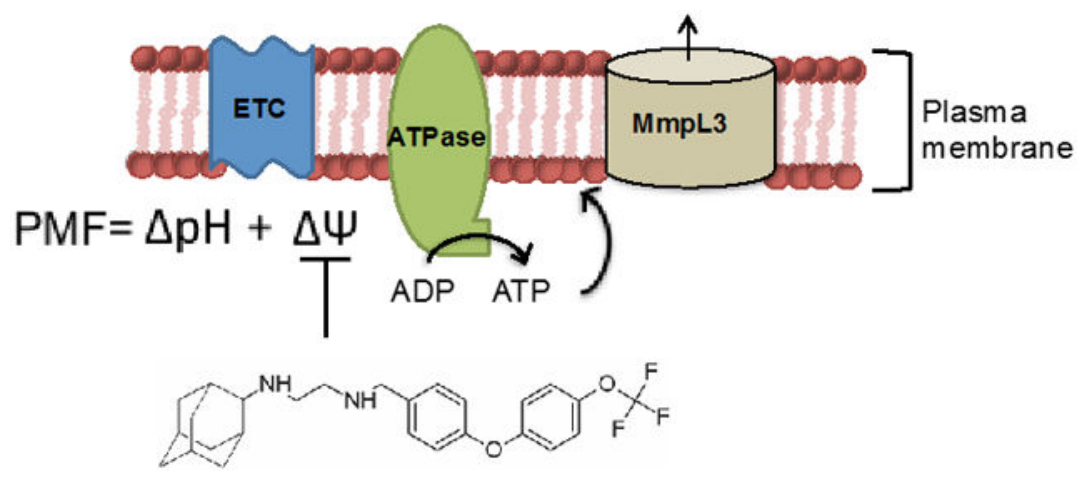

\section{Keywords}

antibiotic; drug development; tuberculosis; proton motive force; MmpL3

Mycobacterium tuberculosis (Mtb), the causative agent of tuberculosis, infects one-third of the world population and caused 1.5 million deaths in 2014. ${ }^{1}$ The current standard therapy for Mtb infection includes rifampicin (RIF, 1), isoniazid (INH, 2), pyrazinamide (PZA, 3), and ethambutol (EMB, 4), of which the latter three remain largely chemically unenhanced since their individual discoveries over 50 years ago. Mtb chemotherapy occurs over a minimum of six months, where non-compliance and known drug toxicities can result in treatment failure. The long duration of Mtb treatment is attributed to heterogeneity in the Mtb population, a fraction of which is thought to enter a non-replicating or quiescent state. This persistent Mtb sub-population are tolerant of both killing by drug therapy and clearance by the immune system. ${ }^{2}$ New therapies should therefore strive to significantly reduce treatment duration and target phenotypically drug-resistant Mtb. In addition, up to 2 million individuals may become infected with multi-drug resistant (MDR)-Mtb strains by $2015 .^{3}$ Therefore, it is critical to develop drugs that function by new mechanisms of action against emerging MDR-Mtb. Efforts to supply the TB drug pipeline include identification of existing clinical antibiotics with efficacy against Mtb as well as discovery of new antimycobacterial core scaffolds. ${ }^{4,5}$

Recently, medicinal chemistry efforts including ours have identified compounds that share structural similarity to EMB, but inhibit Mtb through a new mechanism of action. ${ }^{6} \mathrm{We}$ present the structures of RIF, INH, and EMB in Figure 1, along with developmental intermediate DA5 (5), DA8 (6) the clinical lead SQ109 (7), and our lead compound TBL-140 (8) for comparison. Notably, SQ109 has progressed to Phase II clinical trials in humans. ${ }^{7}$ In characterizing the SQ109 mechanism of action, investigators originally identified the main target as MmpL3, the essential trehalose monomycolate (TMM) transporter. ${ }^{8}$ MmpL3 was also implicated as the target of similar compounds DA5 and DA8 when spontaneous resistant mutants were isolated in screens. In vitro studies revealed that these mutants were cross-resistant to SQ109, supporting a shared mechanism of action. ${ }^{8}$ However upon further investigation, it appears that these putative MmpL3 inhibitors target maintenance of the proton motive force (PMF), which indirectly inhibits PMF-dependent MmpL protein function. ${ }^{9} 10$ Specifically, SQ109 was suggested to be a protonophore at its 
$1 \times \mathrm{MIC}$ in Mycobacterium smegmatis, as it rapidly depleted both the transmembrane potential $(\Delta \Psi)$ and the chemical proton gradient $(\Delta \mathrm{pH})$.

We report herein the development and initial characterization of $\mathbf{8}$. This compound was refined from one of our preliminary anti-malarial compounds identified as a "hit" against Mtb by the Drugs for Neglected Diseases Institute. Compound $\mathbf{8}$ shows antimicrobial activity in vitro against Mtb at low $\mu \mathrm{g} / \mathrm{mL}$ concentrations. To appreciate the antimicrobial activity of $\mathbf{8}$ against $\mathrm{Mtb}$ and assess its potential for further drug development, we determined the ability of the compound to inhibit growth of intracellular Mtb, drug-resistant Mtb strains, and non-growing Mtb populations. We also evaluated the compatibility of $\mathbf{8}$ in combination with current TB therapy drugs. To investigate the molecular target(s) of $\mathbf{8}$, we screened for and isolated 16 independent spontaneous mutants resistant to 8 in Mtb. All 8resistant (TBL-140 ${ }^{\mathrm{R}}$ ) mutants carry nucleotide mutations in $\mathrm{mmpL} 3$ that result in amino acid substitutions. Since recent work suggests that mutations in $m m p L 3$ may not be due to direct inhibition by antimycobacterial compounds, but rather depletion of the PMF, ${ }^{9,10}$ we also evaluated $\Delta \Psi$ and $\Delta \mathrm{pH}$ in mycobacteria treated with $\mathbf{8}$. These studies show that $\mathbf{8}$ targets the mycobacterial $\Delta \Psi$. Consistent with these data, we saw reduced intracellular ATP levels following treatment. Finally, we determined the metabolic stability of $\mathbf{8}$, and found it greatly improved relative to SQ109. Our data show that $\mathbf{8}$ represents a viable therapeutic lead against Mtb.

\section{Results and Discussion}

\section{Targeted structure-activity relationships (SAR) for the core ethylenediamine.}

A compound (12, Supplemental Table 1) from our antimalarial chemical library was characterized as a "soft hit" in an anti-Mtb screen conducted by the Drugs for Neglected Diseases (Geneva, Switzerland). After receiving this information, we noticed there are structural similarities between 12, ethambutol, and SQ109 in that each molecule has a central alkyldiamine flanked by hydrophobic aliphatic groups. Similar compounds within our library were then also screened for MICs to further explore the structure-activity profile. Full synthesis and characterization of 12, 15, 18, 21, 24, 27 and 30 are presented in Supplemental Methods and Supplemental Figures 1 and 2. Compound 12, with a 1,2diaminoethane central unit flanked by bulky adamantyl groups, provided the lowest MIC value in the original testing cascade. Given its resemblance to SQ109 and our desire to replace the geranyl side chain of SQ109 with a proven and metabolically stable bioisostere, the diphenylether side chain, ${ }^{11}$ we prepared $N^{1}$-adamantan-2-yl- $N^{2}$-(4-(4-(trifluoromethoxy)phenoxy)benzyl)ethane-1,2-diamine (TBL-140, 8). The synthesis of $\mathbf{8}$ is shown in Supplemental Figure 2. Based on the impressive low $\mathrm{MIC}_{99}$ value of $1.2 \mu \mathrm{g} / \mathrm{mL}(2.5 \mu \mathrm{M})$ and $\mathrm{MBC}_{99}$ value of $2.3 \mu \mathrm{g} / \mathrm{mL}(5 \mu \mathrm{M})$ observed against $\mathrm{Mtb}$, we further characterized the antibacterial properties of this intriguing molecule.

\section{Compound 8 is effective at clearing intracellular Mtb and drug-resistant strains.}

A critical drug property is the ability of a compound to kill intracellular Mtb without harming the host cell. Treatment with $\mathbf{8}$ at the 1X MIC resulted in two-log reduction in bacterial cfu compared to untreated infected macrophages (Figure 2). This result was similar 
to clinical antibiotic controls and indicates that the compound is able to diffuse into the Mtbinfected cells and reduce bacterial growth and viability. Our assays of cytotoxicity in infected and uninfected THP-1 and Vero cells showed that concentrations of 8 required to reduce Mtb survival were not measurably cytotoxic (Table 1). The selective index (SI), calculated by dividing the cytotoxicity $\mathrm{IC}_{50}$ by the in vitro $\mathrm{H} 37 \mathrm{Rv}$ MIC, of $\mathbf{8}$ is 3.8 and 3.4 in the transformed THP-1 and Vero cell lines (Table 1). The SI for 8 in normal untransformed HFF cells was 16.1. The SI for SQ109 in Vero cells was reported previously for SQ109 as 16.7. ${ }^{12}$ When we performed cytotoxicity assays on HFF cells with SQ109 alongside 8, the SI for SQ109 was 21.4. Therefore, the two compounds have comparable cytotoxicity in vitro, although these results do not address the potential for cytotoxicity upon drug metabolism.

Other hurdles for any new antibiotic to be used for treatment of tuberculosis are its efficacy in combination with other antimicrobials and against drug-resistant Mtb strains. Treatment of Mtb infections with multiple distinct antibiotics reduces the risk of spontaneous emergence of drug resistance. We combined $\mathbf{8}$ with RIF, INH, or EMB in fixed combinations relative to their independent in vitro MICs. In both M. tuberculosis H37Rv and M. bovis BCG, we observed no significant positive or negative interactions between $\mathbf{8}$ and the clinical antibiotics (Figure 3 ). This suggests that $\mathbf{8}$ could be used in combination with existing clinical treatments without adverse effects on potency. We tested $\mathbf{8}$ against both RIF- and INH- resistant Mtb isolates and their corresponding wild-type strain. In each case, $\mathbf{8}$ performed as well against the drug resistant strain as the drug-susceptible counterpart (Table 2). These data also indicate that the mechanism of action for $\mathbf{8}$ does not overlap with these front-line clinical antibiotics.

\section{Compound 8 kills non-replicating Mtb.}

Tuberculosis infections are composed of metabolically heterogeneous populations, including actively dividing and non-growing bacteria that are phenotypically-resistant to front-line antibiotics. ${ }^{2}$ This contributes to the long duration of Mtb chemotherapy. To model nonreplicating, drug-tolerant Mtb, we starved bacteria in PBS and then treated with clinical antibiotics or 8 (Figure 4). Mtb starved for 2-4 weeks are tolerant of RIF, INH, and EMB at the $10 \times$ MIC for actively growing Mtb. However, non-replicating Mtb are sensitive to treatment with 8 and demonstrate a 3-log reduction in viability. This suggests there is a difference in the mechanism of action of these clinical therapeutic agents and $\mathbf{8}$, whereby current antibiotics target processes more essential to growth and $\mathbf{8}$ also targets critical functions required during non-replicating states.

\section{MmpL3 is involved in resistance to 8.}

To identify a target for $\mathbf{8}$, we performed a spontaneous mutant screen on Mtb H37Rv. At the same time, we screened for mutants resistant to DA5, a structurally similar compound that was reported to inhibit MmpL3 ${ }^{8}$ At the solid media $2 \times \mathrm{MIC}$, we acquired 2.07 resistant mutants per $10^{9}$ generations for 8 and 3.88 per $10^{8}$ generations for DA5. In bacteria, single nucleotide mutations occur on average at a rate of 1 per $10^{9}$ generations, ${ }^{13}$ which is consistent with the rate of acquired mutants in our screens. The expected number of 
resistance-conferring events in the parallel cultures was calculated to be 2.191 and 0.227 for the DA5 and $\mathbf{8}$ screens, respectively.

Because the DA5 structure has some similarity to 8 , we evaluated the MIC of the acquired DA $5^{\mathrm{R}}$ and TBL- $140^{\mathrm{R}}$ isolates to these two compounds and found cross-resistance, whereby DA $5^{\mathrm{R}}$ isolates have improved $\mathbf{8}$ resistance and vice-versa. Since mutations in mmpL3 confer resistance to DA5, we sequenced the $m m p L 3$ gene of all the mutants. In total, we identified 16 independent mutants with mutations in $m m p L 3$ that conferred resistance to both DA5 and 8 (Table 3). Between the DA5 ${ }^{\mathrm{R}}$ and TBL- $140^{\mathrm{R}}$ isolates, we observed 12 different nucleotide mutations that result in 11 amino acid substitutions in the $m m p L 3$ gene. Other mutations are certainly present within our screen populations, but the mutations conferring resistance are likely occurring only a single time within cells that produced resistant colonies when plated. In every isolate obtained, a single nucleotide mutation in the $m m p L 3$ gene was observed. We isolated the A700T and L567P point substitutions in MmpL3 that were previously identified as conferring resistance to DA5 and DA8. ${ }^{8}$ We also obtained new MmpL3-resistance alleles. Mutations in $m m p L 3$ also confer resistance to SQ109. ${ }^{8}$ The TBL- $140^{\mathrm{R}}$ isolates also had an increased MIC for SQ109, resulting in increases in the MIC from $1.56 \mu \mathrm{g} / \mathrm{mL}$ in wild-type H37Rv to 6.25 and $12.5 \mu \mathrm{g} / \mathrm{mL}$ in the Leu567Pro and Val643Met substituted MmpL3 isolates, respectively. In contrast, the DA $5^{\mathrm{R}}$ and TBL- $140^{\mathrm{R}}$ strains had identical susceptibility as wild-type H37Rv to RIF, INH, and EMB. Combined, these data suggest that 8, SQ109, and DA5 inhibit Mtb through a similar mechanism that is distinct from the current front-line therapies tested.

To better understand the significance of the mutations identified, we generated an in silico model of MmpL3 (Figure 5) and Ramachandran plot to validate its quality (Supplemental Figure 3). We observe residues substituted in resistant mutants located in the center of the transmembrane domain, as well as alpha helices that contact this pocket. It has been suggested that the impacted transmembrane helices $(4,10,11$, and 12) contribute to the utilization of the PMF. ${ }^{14,15}$ Therefore, the altered residues likely allow MmpL3 function to compensate for a disruption in the PMF strength.

\section{Compound 8 affects mycobacterial PMF resulting in decreased intracellular ATP.}

Recent reports suggest that inhibitors that appear to target MmpL3 based upon genetic analysis of resistant mutants actually impair MmpL3 function through an indirect mechanism involving PMF depletion. ${ }^{9}, 10$ Specifically, Li and colleagues showed that SQ109 at its $1 \times$ MIC behaved like a protonophore in M. smegmatis. Since $\mathbf{8}$ shares the core ethylenediamine with SQ109, we tested 8 for effects on the PMF components $\Delta \Psi$ or $\Delta \mathrm{pH}$ by labeling fast-growing $M$. smegmatis $\mathrm{mc}^{2} 155$ (MIC=9.21 $\mu \mathrm{g} / \mathrm{mL}, 20 \mu \mathrm{M}$ ) and slow-growing M. bovis $\mathrm{BCG}(\mathrm{MIC}=575 \mathrm{ng} / \mathrm{mL}, 1.25 \mu \mathrm{M})$ with $\mathrm{DiOC}_{2}$ or CMFDA, respectively. $\mathrm{DiOC}_{2}$ associates with the inner membrane in bacteria when the PMF is present, causing stacking of the $\mathrm{DiOC}_{2}$ molecules and a shift in fluorescence emission. CMFDA has a pH-sensitive and insensitive fluorescence excitation, allowing for measurement of the bacterial cytoplasmic $\mathrm{pH}$ through the ratio of fluorescence intensity for the two excitation wavelengths.

When M. smegmatis was treated with $1 \times \mathrm{MIC} 8$ or higher, the red / green fluorescence ratio of $\mathrm{DiOC}_{2}$ dropped within 15 minutes, indicating a rapid depletion of $\Delta \Psi$ (Figure $6 \mathrm{~A}$ ). When 
M. smegmatis was treated with 8 at its $1 \times$ or $4 \times \mathrm{MIC}$, the intrabacterial $\mathrm{pH}$ remained near $\mathrm{pH}$ 7 unlike the CCCP control, which equilibrated rapidly to the external pH 5.5 (Figure 6B). In M. bovis BCG, treatment with 8 at its $20 \times \mathrm{MIC}$, but not at the $1 \times \mathrm{MIC}$ resulted in rapid depletion of $\Delta \Psi$ (Figure 6C). None of the concentrations tested reduced $\Delta \mathrm{pH}$ (Figure 6D). Overall, these data show 8 impacts the $\Delta \Psi$ component of the PMF, which is consistent with a target in the ETC. Similarly, treatment of M. bovis BCG with higher concentrations of SQ109 will rapidly deplete $\Delta \Psi$ but not the $\Delta \mathrm{pH}$ (Supplemental Figure 4).

Loss of PMF will reduce the ability of bacteria to generate ATP via the $\mathrm{F}_{0} \mathrm{~F}_{1}$ ATP synthase. We measured intracellular ATP levels following treatment with $\mathbf{8}$. Using this approach, we found that 8 reduces intracellular ATP in a dose-dependent manner (Figure 7). The impact of 8 on ATP levels was on the same order as our positive control, the $\mathrm{F}_{0} \mathrm{~F}_{1}$ ATP synthase inhibitor N,N'-dicyclohexylcarbo-diimide (DCCD). Non-replicating persistent Mtb maintain the same $\Delta \Psi$ as growing cells, ${ }^{16}$ and $\mathbf{8}$ is effective against both replicating and dormant populations. Combined, these data suggest that the target of $\mathbf{8}$ could be a component of the ETC. Future biochemical and genetic approaches will be focused on identifying this target.

Compounds that target the PMF may have general antibacterial activity. To this end, we determined the $\mathrm{IC}_{50}$ of $\mathbf{8}$ against a small number of other bacteria. We found that $\mathbf{8}$ was effective against selected Gram-negative and Gram-positive species: 8 had an $\mathrm{IC}_{50}$ of 6.25 $\mu \mathrm{g} / \mathrm{mL}$ against Escherichia coli, Staphylococcus aureus, and Bacillus subtilis. The $\mathrm{IC}_{50}$ against Pseudomonas aeruginosa was $12.5 \mu \mathrm{g} / \mathrm{mL}$. These results are consistent with the target of $\mathbf{8}$ being the PMF or another well-conserved protein.

\section{Compound 8 has improved drug properties over SQ109.}

One goal at the outset of this study was to develop a compound that had improved metabolic stability relative to SQ109, another diamine molecule being considered as a TB therapeutic. We therefore directly compared the in vitro metabolic stability of $\mathbf{8}$ and SQ109 in the presence of $1 \mathrm{mg} / \mathrm{mL}$ human liver microsomes. Table 4 provides the predicted hepatic extraction ratio $(\mathrm{EH})$ for $\mathbf{8}, \mathrm{SQ109}$, and midazolam, a high extraction ratio control. The in vitro half-life $\left(\mathrm{t}_{1 / 2}\right)$ of $\mathbf{8}$ was 231 minutes, compared to 2.97 minutes for SQ109 and is reflected in the large difference in the intrinsic clearance $\left(\mathrm{CL}_{\text {int }}\right)$ for $8(3.47 \mathrm{~mL} / \mathrm{min})$ vs SQ109 (269 $\mathrm{mL} / \mathrm{min})$. We also compared the in vitro metabolic stability of 8 and SQ109 in the presence of $1 \mathrm{mg} / \mathrm{mL}$ mouse liver microsomes. The in vitro half-life $\left(\mathrm{t}_{1 / 2}\right)$ of $\mathbf{8}$ was 24.14 minutes, compared to 2.44 minutes for SQ109 (Table 4). Others have also observed that SQ109 is rapidly metabolized. ${ }^{17}$ Since SQ109 is rapidly metabolized in vitro, the in vivo mechanism may be different than what has been proposed for the parent drug based on in vitro analysis. Independent analysis of $\mathbf{8}$ by Cyprotex using primary human hepatocytes yielded consistent results: The half-life of $\mathbf{8}$ was 190 minutes. In comparison, the half-life of the low and high clearance controls (warfarin and verapamil, respectively) were $>480$ and 50.5. These data indicate that $\mathbf{8}$ has excellent metabolic stability and support further investigation of the molecule to further enhance selectivity toward the pathogen.

In this report we describe 8 , an $N^{1}$-adamantan-2-yl- $N^{2}$-(4-(4-(trifluoromethoxy)phenoxy)benzyl)ethane-1,2-diamine molecule with antimycobacterial activity. We 
show that $8(\mathrm{MIC}=1.2 \mu \mathrm{g} / \mathrm{mL}$ ) shows similar activity and potency to SQ109 (MIC=1.6 $\mu \mathrm{g}$ / $\mathrm{mL}$ ), but represents a new chemical space that does not require the geranyl moiety. ${ }^{7-9}$ The chemical changes present in $\mathbf{8}$ result in a more chemically stable drug in the presence of liver enzymes. The compound is effective at killing intracellular Mtb in an in vitro infection model and inhibits growth of drug-resistant $\mathrm{Mtb}$. Compound $\mathbf{8}$ can be used effectively in combination with current clinical antibiotics and shows potential against phenotypicallyresistant, non-growing Mtb populations. In the context of a tuberculosis infection where the Mtb population is heterogeneous and includes a population of persistent organisms, the addition of $\mathbf{8}$ may reduce the required treatment time in the presence of existing drug therapy.

Mechanistically, the effects of $\mathbf{8}$ on mycobacteria are consistent with a block of the ETC. At concentrations where $\mathbf{8}$ affected the PMF, only $\Delta \Psi$ was perturbed; 8 did not equilibrate $\Delta \mathrm{pH}$ at any concentration tested. With regards to the spontaneous point mutations in $m m p L 3$ that confer 8 resistance, we believe these are consistent with compensatory mutations that allow MmpL3 to function despite a disruption to the strength of the PMF. Quiescent Mtb maintain their membrane potential suggesting that the development of compounds that target respiratory functions will be effective against replicating and non-replicating organisms. ${ }^{16}$ Based upon our results, $\mathbf{8}$ may be generally categorized as such a compound as it is effective against Mtb in broth culture, infected macrophages and an in vitro model of dormancy.

It is intriguing that $1 \times$ MIC 8 was sufficient for rapid loss of $\Delta \Psi$ in $M$. smegmatis, but not $M$. bovis BCG. There may be species-dependent differences in the maintenance or recovery of the PMF, or the effects on $\Delta \Psi$ may be due to different targets between these two strains. It is worth noting that the MmpL3 protein is present in both the fast-growing M. smegmatis and slow-growing $M$. bovis $\mathrm{BCG}$, but only the $M$. bovis sequence is identical to Mtb. The MmpL3 sequence from M. smegmatis has amino acid differences in 6 of the 11 amino acid residues identified as substituted in TBL- $140^{\mathrm{R}}$ Mtb. Further work is necessary to define the direct and indirect targets involved in the $\mathbf{8}$ mechanism of action in mycobacteria species as there are likely multiple indirect targets, including MmpL3, due to effects on the PMF.

\section{Methods}

\section{Chemical synthesis and analysis.}

All synthetic procedures and analysis of compound properties is reported in the Supplemental Materials.

\section{Strains, cell culture, media, and antimicrobial agent preparation.}

Mycobacterium smegmatis mc $^{2} 155$, Mycobacterium bovis BCG, Mtb H37Rv, Mtb T 21

(INH-sensitive), Mtb T 27 (INH-resistant), Mtb Beijing 26 (RIF-sensitive), Mtb Beijing 22

(RIF-resistant), Mtb Haarlem 23 (RIF-sensitive), Mtb Haarlem 9 (RIF-resistant) were grown in Middlebrook 7H9 liquid broth (BD Difco ${ }^{\mathrm{TM}}$ ) containing $0.05 \%$ Tween 80. Isogenic drugsusceptible and drug-resistant isolates were confirmed as parent-mutant pairs by spoligotyping and insertion sequence 6110 (IS6110) restriction fragment length polymorphism (RFLP) as described previously. ${ }^{18}$ We grew E. coli DH5-a, B. subtilis 
CU1065, S. aureus ATCC 29213 and P. aeruginosa ATCC 27853 in Luria broth. All mycobacterial and mammalian cell cultures were incubated at $37{ }^{\circ} \mathrm{C}$ with $5.5 \% \mathrm{CO}_{2}$. We measured colony forming units (cfu) by dilution of mycobacterial strains in phosphate buffered saline (PBS) and plating onto 7H10 agar (BD Difco ${ }^{\mathrm{TM}}$ ). We cultured THP-1 cells in 90\% RPMI1640 (HyClone), 10\% heat-inactivated FBS (Sigma), supplemented with $2 \mathrm{mM}$ L-glutamine (HyClone). To differentiate THP-1 cells, 50 nM phorbol-12-myristate-13acetate (PMA) was added for $48 \mathrm{~h}$. We maintained Henle cell cultures in 90\% Dulbecco's Modified Eagle Medium (DMEM), 10\% heat-inactivated FBS, supplemented with $2 \mathrm{mM} \mathrm{L-}$ glutamine, and 1X MEM non-essential amino acids (Gibco). We grew Vero cells in $90 \%$ DMEM, 10\% heat-inactivated FBS, supplemented with $2 \mathrm{mM} \mathrm{L}$-glutamine, $100 \mathrm{U} / \mathrm{mL}$ penicillin, $100 \mu \mathrm{g} / \mathrm{mL}$ streptomycin, and 1X MEM non-essential amino acids. We grew normal human foreskin fibroblast (HFF) cells in DMEM supplemented with $10 \%$ heatinactivated fetal bovine serum, $2 \mathrm{mM} \mathrm{L}$-glutamine, $100 \mathrm{U} / \mathrm{mL}$ penicillin, $100 \mu \mathrm{g} / \mathrm{mL}$ streptomycin. Aliquots of 8, SQ109 (MedChem Express), DA5 (ChemBridge), rifampacin (Sigma), isoniazid (Acros Organics), ethambutol (MP Biomedicals), and carbonyl cyanide m-chlorophenylhydrazone (CCCP) (Sigma) were prepared in either DMSO or $\mathrm{dH}_{2} \mathrm{O}$ every 6 months and stored at $-20^{\circ} \mathrm{C}$.

\section{Antimicrobial susceptibility testing.}

Culture media containing antibiotics in 2-fold dilution series were prepared and transferred to round-bottom plates for evaluation of drug susceptibility of mycobacteria. We inoculated plates with $\sim 5 \times 10^{5} \mathrm{cfu} / \mathrm{mL}$ exponential phase cultures and plated the inoculum on $7 \mathrm{H} 10$ agar to confirm initial cfus. We visually established $\mathrm{MIC}_{99}$ endpoints after $1 \mathrm{~d}$ or $5 \mathrm{~d}$ incubation for M. smegmatis or M. bovis and Mtb, respectively, by finding the lowest concentration at which there was an absence of bacterial pellets. To confirm endpoints and measure the minimal bactericidal concentration (MBC), we plated dilutions of the control cultures and inhibited cultures to enumerate cfu after treatment. For solid macrodilution MIC testing, we prepared dilutions of antibiotics in solvent in 2-fold dilution series. We distributed the dilution series of the antibiotic to plastic dishes and added a set volume of $7 \mathrm{H} 10$ agar cooled to $60^{\circ} \mathrm{C}$. $\mathrm{IC}_{50}$ determination against E. coli DH5-a, B. subtilis CU1065, $S$. aureus ATCC 29213 and $P$. aeruginosa ATCC 27853 was performed essentially as described. ${ }^{9}$

\section{Fractional inhibitory concentration index ( $\mathrm{FICl})$ determination.}

We performed MIC testing of drugs in 2-fold dilutions in isolation and in fixed combinations of 4:1, 3:2, 2:3, or 1:4 relative to their previously established MICs. We then observed the endpoints that resulted in growth inhibition and determined the concentration of each antibiotic in the mixture. The concentration of the individual components was divided by their MIC in isolation to calculate their fractional inhibitory concentration (FIC). The sum of these fractions was used to determine the FIC index for each fixed drug combination. We interpreted the FICI endpoints as additive between 0.5-4, synergistic when $₫ 0.5$, and antagonistic when $>4 .{ }^{19}$ 


\section{PBS - starved Mtb.}

We starved Mtb of nutrients by suspension in PBS with modifications to a previously published protocol. ${ }^{20}$ We pelleted exponential phase Mtb to prepare cultures at a final density of $1 \times 10^{8}$ cells $/ \mathrm{mL}$. Mtb was washed twice in PBS and then resuspended in PBS. Dilutions of the final bacterial suspension were plated for enumeration of the starting inoculum. Cultures were incubated in flasks with filter caps at $37{ }^{\circ} \mathrm{C}$ with $5.5 \% \mathrm{CO}_{2}$ for durations of 2 or 4 weeks before treatment.

\section{Spontaneous mutant screening.}

We screened for resistant mutants using a standard fluctuation assay. ${ }^{21}$ We inoculated 200 $\mu \mathrm{L} 7 \mathrm{H} 9$ media with $2 \times 10^{3}$ cells $/ \mathrm{mL}$ or $2 \mathrm{~mL} 7 \mathrm{H} 9$ media with $1 \times 10^{4}$ cells $/ \mathrm{mL}$ H37Rv for the DA5 and 8 screens, respectively. After $17 \mathrm{~d}$ growth, 48 independently grown cultures were spread on 3-mL agar pads containing $20 \mu \mathrm{M}$ DA5 in 12-well plates. For the 8 screen, we plated 20 cultures on $20-\mathrm{mL}$ agar containing $5 \mu \mathrm{M} 8$. Dilutions of cultures grown in parallel were plated to enumerate the final cfu for each screen. To determine the rate of spontaneous mutation in the screen, we counted the resistant colonies present on each plate and input the values into the FALCOR calculator. ${ }^{22}$ Two colonies from each well or plate containing colonies were transferred to fresh solid media containing antibiotic for isolation. We inoculated $7 \mathrm{H} 9$ media with isolates for storage at $-80^{\circ} \mathrm{C}$ and preparation of cultures for MIC measurements.

\section{Amplification and sequencing of $\mathrm{mmpL3}$.}

Genomic DNA was purified from the parent strain H37Rv and 8-resistant isolates. We amplified $m m p L 3$ in $50 \mu \mathrm{L}$ reactions using $25 \mathrm{ng}$ of purified genomic DNA as template using Qiagen Taq following the manufacturer recommendations using the previously designed forward primer of 5'-GACGTGTGTGACAACCAAA-3' and reverse primer of 5'AGGTGCTGCTGGGCGGGAA-3 ${ }^{\prime} \cdot{ }^{23}$ After a 3 minute denaturing step at $95^{\circ} \mathrm{C}$, we cycled reactions a total of 30 times through the following: 1 minute denature at $95{ }^{\circ} \mathrm{C}, 1$ minute annealing at $55^{\circ} \mathrm{C}$, and 3 minutes extension at $72{ }^{\circ} \mathrm{C}$. We used a final elongation at $72{ }^{\circ} \mathrm{C}$ of 10 minutes. The $m m p L 3$ PCR product was gel purified using a Zymoclean Gel DNA recovery kit and sequenced.

\section{Compound cytotoxicity evaluation.}

THP- 1 cells were seeded in 96-well flat-bottom plates at a density of $1 \times 10^{4}$ cells/well with PMA for $48 \mathrm{~h}$ before addition of solvent controls or antibiotics. Vero were seeded in 96 -well flat-bottom plates at a density of $2.5 \times 10^{3}$ cells/well and added solvent controls or antibiotics after $4 \mathrm{~h}$. We incubated cells with treatment for $18 \mathrm{~h}$ (THP-1 and Vero) or $48 \mathrm{~h}$ (HFF) before labeling cells with Alamar Blue to determine percent survival. We used the fluorescence of Alamar Blue excited at $510 \mathrm{~nm}$ and read at $590 \mathrm{~nm}$ to determine the inhibitory concentration that resulted in $50 \%$ mammalian cell loss $\left(\mathrm{IC}_{50}\right)$.

\section{Cell culture-based Mtb infection.}

We seeded THP- 1 cells at a density of $1 \times 10^{4} /$ well in 96 -well flat bottom plates in medium containing $30 \mathrm{ng} / \mathrm{mL}$ PMA for $48 \mathrm{~h}$. Differentiated THP-1 cells were infected at an MOI of 
1:1 for $1 \mathrm{~h}$ then washed with media to remove extracellular bacteria. Antibiotics were added to infected THP-1 cells at multiples of their in vitro MICs against H37Rv. After 5d incubation, THP- 1 cells were lysed with PBS $0.1 \%$ Tween, serially diluted and plated on $7 \mathrm{H} 10$ agar plates to determine the cfu of surviving H37Rv.

\section{In silico modeling of MmpL3.}

A model of the MmpL3 protein structure was generated by homology to the resistance/ nodulation/cell division family proteins AcrB (PDB 4dx5), MexB (PDB 3w9i), and MtrD (PDB 4mt1) from Escherichia coli, Pseudomonas aeruginosa, and Neisseria gonorrhoeae, respectively. ${ }^{24-26}$ The MmpL3 amino acid sequence was aligned first with HHpred and the alignments submitted to the Modeller server available through the Bioinformatics Toolkit from the Max-Planck Institute for Developmental Biology. ${ }^{27}$ Images of the MmpL3 model were prepared using PyMOL Molecular Graphics System, v1.3 (Schrodinger LLC). To validate the fidelity of the model, we checked the number of amino acids within the MmpL3 core that fall outside of allowable ranges, as determined by RAMPAGE Ramachandran plot analysis. ${ }^{28}$ Within the core of MmpL3, the number of residues in outlier regions is $3.8 \%$ and is considered to be acceptable for an in silico model.

\section{Measurement of the mycobacterial transmembrane potential $(\Delta \Psi)$.}

We labeled mycobacteria with the Nerstian dye diethyloxacarbocyanine iodide $\left(\mathrm{DiOC}_{2}\right.$, Molecular Probes, Eugene, OR) to measure $\Delta \Psi$. We labeled M. smegmatis $\mathrm{mc}^{2} 155$ and $M$. bovis BCG with $30 \mu \mathrm{M} \mathrm{DiOC}{ }_{2}$ in PBS supplemented with $50 \mathrm{mM} \mathrm{KCl}, \mathrm{pH} 7.2$ for 15 minutes, followed by two washes with buffer and resuspension in buffer at $\mathrm{OD}_{600}=0.2$. We treated suspensions in triplicate with DMSO, the in vitro $1 \times$ MIC of the protonophore CCCP, or $1 \times$ MIC or multiples of the in vitro MIC for $\mathbf{8}$ and SQ109 for the bacteria in the experiment. We determined the red/green fluorescence ratio of $\mathrm{DiOC}_{2}$ emission using a Gemini EM fluorescence microplate reader (Molecular Devices, Sunnyvale, CA). $\mathrm{DiOC}_{2}$ was excited at $485 \mathrm{~nm}$ and emission was measured at 610 and $515 \mathrm{~nm}$. The ratio of fluorescence intensity at $610 \mathrm{~nm} / 515 \mathrm{~nm}$ is relative to the strength of $\Delta \Psi$.

\section{Measurement of the mycobacterial chemical proton potential $(\Delta \mathrm{pH})$.}

We labeled either M. smegmatis $\mathrm{mc}^{2} 155$ or M. bovis BCG with 5-chloromethylfluorescein diacetate (CMFDA, Molecular Probes, Eugene, OR) in PBS pH 7.0. We labeled mycobacteria with $0.5 \mathrm{mg} / \mathrm{mL}$ CMFDA in buffer for 10 minutes, followed by two washes with buffer $\mathrm{pH} 7.0$ and resuspension in PBS buffer at $\mathrm{pH}$ 5.5. We treated cells in triplicate with DMSO, $1 \times$ MIC concentrations of the protonophore CCCP, or $1 \times \mathrm{MIC}$ or multiples of the MIC for 8 and SQ109. We calibrated the cytoplasmic pH measurement by treatment of labeled cells with $10 \mu \mathrm{M}$ nigericin in $0.1 \mathrm{M}$ potassium acetate for $\mathrm{pH} 5$ and 5.5 , or $0.1 \mathrm{M}$ PIPES for $\mathrm{pH} \mathrm{6,6.5,7,} \mathrm{and} \mathrm{7.5.} \mathrm{We} \mathrm{measured} \mathrm{fluorescence} \mathrm{emission} \mathrm{at} 520 \mathrm{~nm}$ with excitation of 440 and $490 \mathrm{~nm}$. We determined the ratio of fluorescence intensity excited at $490 \mathrm{~nm} / 440 \mathrm{~nm}$ to calculate the $\mathrm{pH}$ of the bacterial cytoplasm. 


\section{Measurement of intracellular ATP.}

Briefly, M. bovis BCG was treated overnight with the indicated concentrations of compound. Cells were disrupted by bead-beating with $200-300 \mathrm{~mm}$ glass beads in an extraction buffer (100 mM Tris, 4 mM EDTA, pH 7.5) using 3 pulses of 1 min each. ATP concentration was quantified using the Molecular Probes' ATP Determination Kit.

\section{Measurement of metabolic stability.}

The degradation of compounds in the presence of human liver microsomes was used to predict the hepatic extraction ratio and characterize the metabolic stability profile of $\mathbf{8}$ versus SQ109 and a high extraction ratio control drug, midazolam. Briefly, human liver microsomes $(1 \mathrm{mg} / \mathrm{mL})$ were incubated with $1 \mu \mathrm{M}$ compound in the presence of an NADPH regenerating system. Aliquots were removed at $\mathrm{t}=0,5,10,20$, and $40 \mathrm{~min}$ and quenched with acetonitrile containing internal standard. The amount of parent compound remaining in the supernatant was assessed by LC-MS/MS. The first-order rate constant for substrate depletion was determined from a linear least squares fit of the natural $\log$ of the percent parent compound remaining versus time. The rate constant was used to calculate the in vitro intrinsic clearance value $\left(\mathrm{CL}_{\text {int }}\right)$ and the predicted in vivo hepatic extraction ratio $(\mathrm{EH})$ as described by Obach et al. ${ }^{29}$ The compounds were stable in the absence of the regenerating system. Analysis of metabolic breakdown of $\mathbf{8}$ by human hepatocytes was performed by Cyprotex (Watertown, MA). Briefly, cryopreserved human hepatocytes were thawed, viable cells counted, and equilibrated. Compound $\mathbf{8}$ was added to $10^{6}$ cells at a final concentration of $1 \mu \mathrm{M}$, and the cell suspension was incubated at $37^{\circ} \mathrm{C}$. Samples were removed over a 120 minute time course and mixed with an equal volume of ice-cold stop solution (methanol containing internal analytical standards). Stopped reactions were incubated at least ten minutes on ice and analyzed by LC-MS/MS to quantitate the remaining parent.

\section{Supplementary Material}

Refer to Web version on PubMed Central for supplementary material.

\section{Acknowledgements}

MHF was supported by a Medical Research Foundation of Oregon Early Clinical Investigator grant and NIH T32 AI 007472. This project was supported with funds from the US Dept. of Veterans Affairs (MKR). The National Science Foundation (Grant 0741993) supported the purchase of the LTQ-Orbitrap Discovery MS system used for analysis of our samples. We thank Dr. Karen Dobos at Colorado State University for sharing the clinical susceptible and mono-resistant Mtb pairs.

\section{Abbreviations}

$\begin{array}{ll}\text { (RIF) } & \text { rifampicin } \\ (\text { INH }) & \text { isoniazid } \\ (\text { EMB }) & \text { ethambutol } \\ (\text { MIC 99) } & \text { minimal inhibitory concentration } \\ (\text { PMF }) & \text { proton motive force }\end{array}$


(CMFDA) 5-chloromethylfluorescein diacetate

$\left(\mathbf{D i O C}_{2}\right) \quad$ diethyloxacarbocyanine iodide

\section{References}

(1). WHO I Tuberculosis. WHO 2015.

(2). Zhang Y; Yew WW; Barer MR Targeting persisters for tuberculosis control. Antimicrob. Agents Chemother. 2012, 56 (5), 2223-2230 DOI: 10.1128/AAC.06288-11. [PubMed: 22391538]

(3). WHO. Fact Sheet MDR-TB.

(4). Favrot L; Ronning DR Targeting the mycobacterial envelope for tuberculosis drug development. Expert Rev Anti Infect Ther 2012, 10 (9), 1023-1036 DOI: 10.1586/eri.12.91. [PubMed: 23106277]

(5). Zumla A; Nahid P; Cole ST Advances in the development of new tuberculosis drugs and treatment regimens. Nat Rev Drug Discov 2013, 12 (5), 388-404 DOI: 10.1038/nrd4001. [PubMed: 23629506]

(6). Lee RE; Protopopova M; Crooks E; Slayden RA; Terrot M; Barry CE Combinatorial lead optimization of [1,2]-diamines based on ethambutol as potential antituberculosis preclinical candidates. J Comb Chem 2003, 5 (2), 172-187 DOI: 10.1021/cc020071p. [PubMed: 12625709]

(7). Sacksteder KA; Protopopova M; Barry CE; Andries K; Nacy CA Discovery and development of SQ109: a new antitubercular drug with a novel mechanism of action. Future Microbiology 2012, 7 (7), 823-837 DOI: 10.2217/fmb.12.56. [PubMed: 22827305]

(8). Tahlan K; Wilson R; Kastrinsky DB; Arora K; Nair V; Fischer E; Barnes SW; Walker JR; Alland D; Barry CE; et al. SQ109 Targets MmpL3, a Membrane Transporter of Trehalose Monomycolate Involved in Mycolic Acid Donation to the Cell Wall Core of Mycobacterium tuberculosis. Antimicrob. Agents Chemother. 2012, 56 (4), 1797-1809 DOI: 10.1128/AAC. 05708-11. [PubMed: 22252828]

(9). Li K; Schurig-Briccio LA; Feng X; Upadhyay A; Pujari V; Lechartier B; Fontes FL; Yang H; Rao G; Zhu W; et al. Multitarget drug discovery for tuberculosis and other infectious diseases. J. Med. Chem. 2014, 57 (7), 3126-3139 DOI: 10.1021/jm500131s. [PubMed: 24568559]

(10). Feng X; Zhu W; Schurig-Briccio LA; Lindert S; Shoen C; Hitchings R; Li J; Wang Y; Baig N; Zhou T; et al. Antiinfectives targeting enzymes and the proton motive force. Proceedings of the National Academy of Sciences 2015, 201521988 DOI: 10.1073/pnas.1521988112.

(11). Nilsen A; Miley GP; Forquer IP; Mather MW; Katneni K; Li Y; Pou S; Pershing AM; Stickles AM; Ryan E; et al. Discovery, Synthesis, and Optimization of Antimalarial 4(1 H)-Quinolone-3Diarylethers. J. Med. Chem. 2014, 57 (9), 3818-3834 DOI: 10.1021/jm500147k. [PubMed: 24720377]

(12). Protopopova M Identification of a new antitubercular drug candidate, SQ109, from a combinatorial library of 1,2-ethylenediamines. J. Antimicrob. Chemother. 2005, 56 (5), 968-974 DOI: 10.1093/jac/dki319. [PubMed: 16172107]

(13). Drake JW A constant rate of spontaneous mutation in DNA-based microbes. Proc. Natl. Acad. Sci. U.S.A. 1991, 88 (16), 7160-7164. [PubMed: 1831267]

(14). Bernut A; Viljoen A; Dupont C; Sapriel G; Blaise M; Bouchier C; Brosch R; de Chastellier C; Herrmann J-L; Kremer L Insights into the smooth-to-rough transitioning in Mycobacterium bolletii unravels a functional Tyr residue conserved in all mycobacterial MmpL family members. Mol. Microbiol. 2015, 99 (5), n/a-n/a DOI: 10.1111/mmi.13283.

(15). Murakami S; Nakashima R; Yamashita E; Yamaguchi A Crystal structure of bacterial multidrug efflux transporter AcrB. Nature 2002, 419 (6907), 587-593 DOI: 10.1038/nature01050. [PubMed: 12374972]

(16). Rao SPS; Alonso S; Rand L; Dick T; Pethe K The protonmotive force is required for maintaining ATP homeostasis and viability of hypoxic, nonreplicating Mycobacterium tuberculosis. Proceedings of the National Academy of Sciences 2008, 105 (33), 11945-11950 DOI: 10.1073/ pnas.0711697105. 
(17). Jia L; Noker PE; Coward L; Gorman GS; Protopopova M; Tomaszewski JE Interspecies pharmacokinetics and in vitro metabolism of SQ109. Br. J. Pharmacol. 2006, 147 (5), 476-485 DOI: 10.1038/sj.bjp.0706650. [PubMed: 16432511]

(18). Bisson GP; Mehaffy C; Broeckling C; Prenni J; Rifat D; Lun DS; Burgos M; Weissman D; Karakousis PC; Dobos K Upregulation of the phthiocerol dimycocerosate biosynthetic pathway by rifampin-resistant, rpoB mutant Mycobacterium tuberculosis. J. Bacteriol. 2012, 194 (23), 6441-6452 DOI: 10.1128/JB.01013-12. [PubMed: 23002228]

(19). Odds FC Synergy, antagonism, and what the chequerboard puts between them. J. Antimicrob. Chemother. 2003, 52 (1), 1-1 DOI: 10.1093/jac/dkg301. [PubMed: 12805255]

(20). Li W; Upadhyay A; Fontes FL; North EJ; Wang Y; Crans DC; Grzegorzewicz AE; Jones V; Franzblau SG; Lee RE; et al. Novel insights into the mechanism of inhibition of MmpL3, a target of multiple pharmacophores in Mycobacterium tuberculosis. Antimicrob. Agents Chemother. 2014, 58 (11), 6413-6423 DOI: 10.1128/AAC.03229-14. [PubMed: 25136022]

(21). Foster PL Methods for determining spontaneous mutation rates. Methods in Enzymology 2006, 409, 195-213 DOI: 10.1016/S0076-6879(05)09012-9. [PubMed: 16793403]

(22). Hall BM; Ma C-X; Liang P; Singh KK Fluctuation analysis CalculatOR: a web tool for the determination of mutation rate using Luria-Delbruck fluctuation analysis. Bioinformatics 2009, 25 (12), 1564-1565 DOI: 10.1093/bioinformatics/btp253. [PubMed: 19369502]

(23). Poce G; Bates RH; Alfonso S; Cocozza M; Porretta GC; Ballell L; Rullas J; Ortega F; De Logu A; Agus E; et al. Improved BM212 MmpL3 inhibitor analogue shows efficacy in acute murine model of tuberculosis infection. PLoS ONE 2013, 8 (2), e56980 DOI: 10.1371/journal.pone. 0056980. [PubMed: 23437287]

(24). Lei H-T; Chou T-H; Su C-C; Bolla JR; Kumar N; Radhakrishnan A; Long F; Delmar JA; Do SV; Rajashankar KR; et al. Crystal structure of the open state of the Neisseria gonorrhoeae MtrE outer membrane channel. PLoS ONE 2014, 9 (6), e97475 DOI: 10.1371/journal.pone.0097475. [PubMed: 24901251]

(25). Nakashima R; Sakurai K; Yamasaki S; Hayashi K; Nagata C; Hoshino K; Onodera Y; Nishino K; Yamaguchi A Structural basis for the inhibition of bacterial multidrug exporters. Nature 2013, 500 (7460), 102-106 DOI: 10.1038/nature12300. [PubMed: 23812586]

(26). Eicher T; Cha H-J; Seeger MA; Brandstätter L; El-Delik J; Bohnert JA; Kern WV; Verrey F; Grütter MG; Diederichs K; et al. Transport of drugs by the multidrug transporter AcrB involves an access and a deep binding pocket that are separated by a switch-loop. Proceedings of the National Academy of Sciences 2012, 109 (15), 5687-5692 DOI: 10.1073/pnas.1114944109.

(27). Biegert A; Mayer C; Remmert M; Söding J; Lupas AN The MPI Bioinformatics Toolkit for protein sequence analysis. Nucleic Acids Res. 2006, 34 (Web Server issue), W335-W339 DOI: 10.1093/nar/gk1217. [PubMed: 16845021]

(28). Lovell SC; Davis IW; Arendall WB; de Bakker PIW; Word JM; Prisant MG; Richardson JS; Richardson DC Structure validation by Calpha geometry: phi,psi and Cbeta deviation. Proteins 2003, 50 (3), 437-450 DOI: 10.1002/prot.10286. [PubMed: 12557186]

(29). Obach RS Prediction of human clearance of twenty-nine drugs from hepatic microsomal intrinsic clearance data: An examination of in vitro half-life approach and nonspecific binding to microsomes. Drug Metab. Dispos. 1999, 27 (11), 1350-1359. [PubMed: 10534321] 


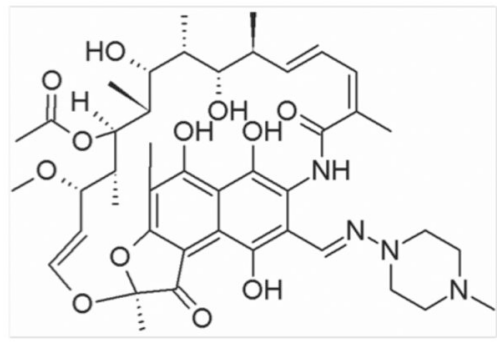

1

Rifampicin (RIF)<smiles>NC(=O)c1cnccn1</smiles>

3

Pyrazinamide (PZA)

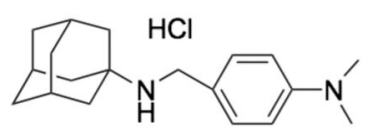

5

DA5<smiles>NNC(=O)c1ccncc1</smiles>

2

Isoniazid (INH)<smiles>CCC(CO)NCCNC(CC)CO</smiles>

Ethambutol (EMB)<smiles>CC(C)=CCC/C(C)=C/CNCCNC1C2CC3CC(C2)C3C1O[Na]</smiles><smiles>O=C(CC12CC3CC(CC(C3)C1)C2)Nc1ccc(F)c(Cl)c1</smiles>

6

DA8

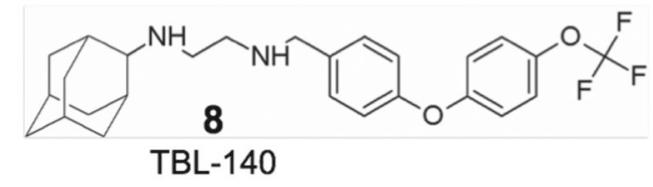

Figure 1 -. Chemical structures of compounds referred to in this study.

Rifampicin, isoniazid, pyrazinamide and ethambutol are first-line antibiotics used in TB treatment. DA5 and DA8 are antibiotic development intermediates and SQ109 is currently being evaluated in clinical trials. TBL-140 is the lead compound characterized in this study. 


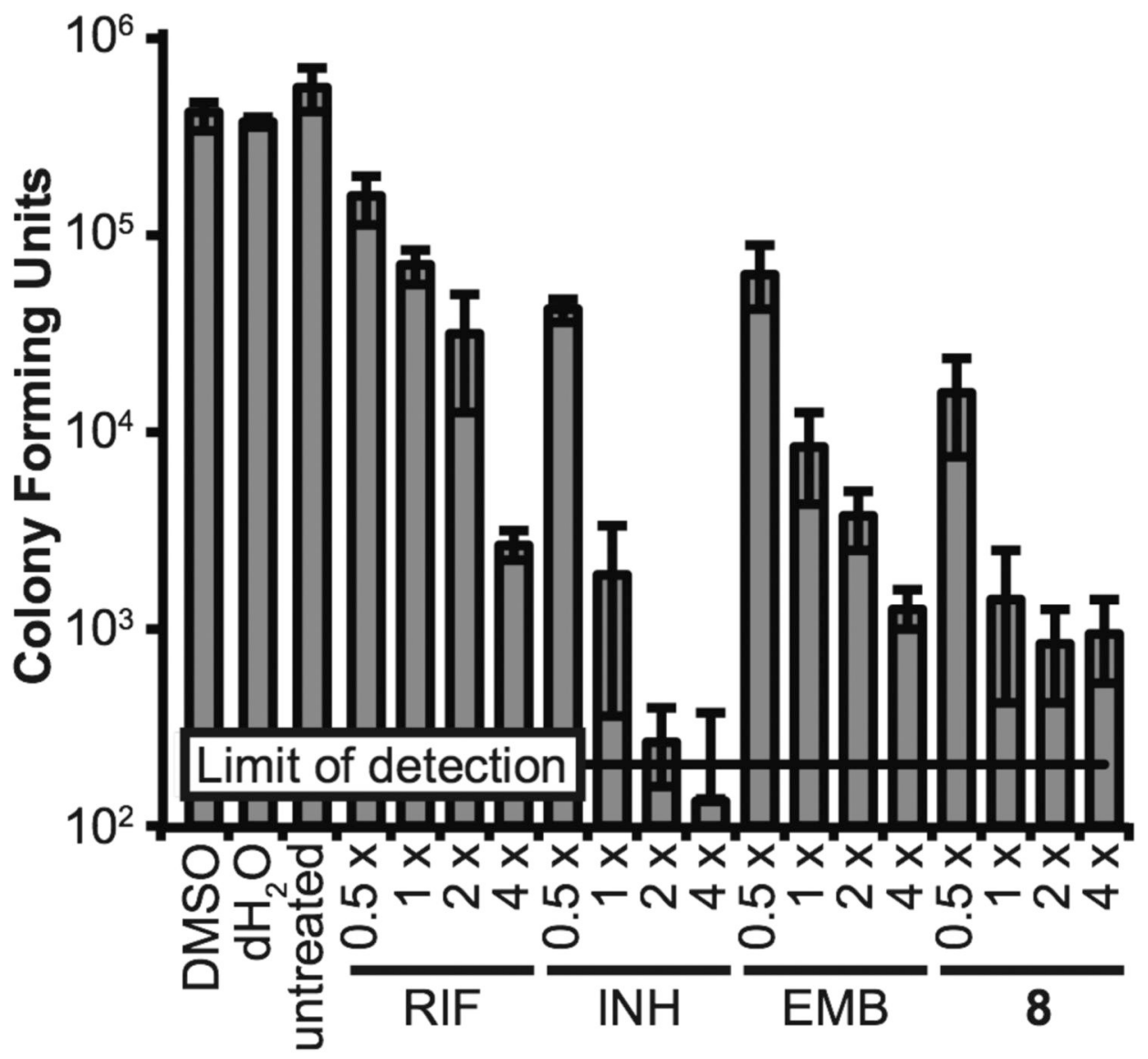

Figure 2 -. Treatment with 8 reduced intracellular Mtb survival.

We infected THP-1 cells with Mtb H37Rv and then treated with either solvent controls, antibiotics (RIF $1 \times \mathrm{MIC}=25 \mathrm{ng} / \mathrm{mL}$, INH $1 \times \mathrm{MIC}=50 \mathrm{ng} / \mathrm{mL}, \mathrm{EMB} 1 \times \mathrm{MIC}=2 \mu \mathrm{g} / \mathrm{mL}$ ), or 8 for $5 \mathrm{~d}$ in concentrations relative to their in vitro MICs. Compound 8 reduced Mtb burden as well as clinical antibiotics. A single representative replicate from three independent experiments is shown. 
a
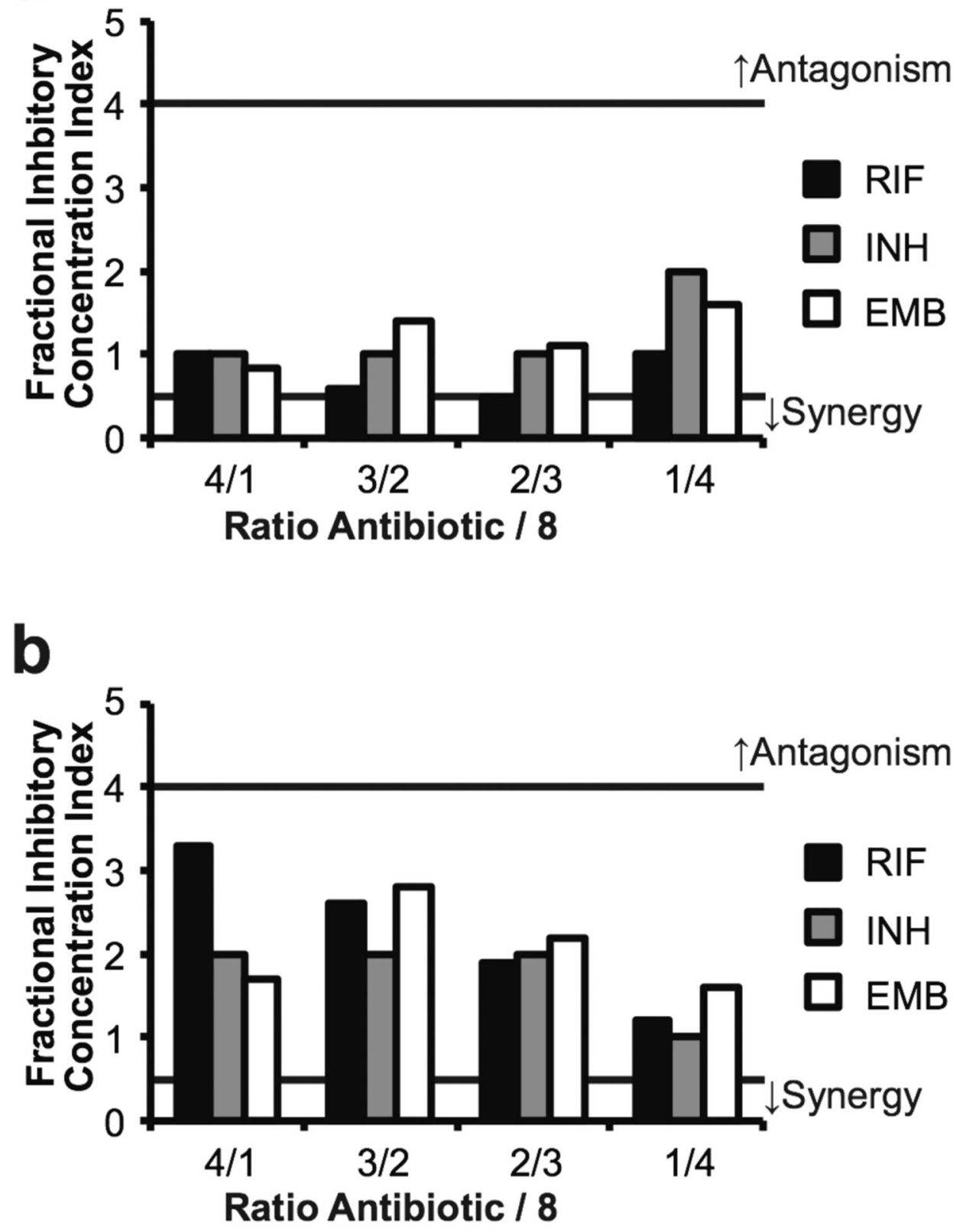

Figure 3 -. Interaction of 8 with clinical antibiotics.

We tested the Mtb growth inhibitory activity of clinical antibiotics RIF, INH, and EMB in combination with $\mathbf{8}$ at fixed ratios, relative to their in vitro MICs alone in A) M. tuberculosis H37Rv and B) M. bovis BCG. The fractional inhibitory concentration index indicates the potency of the combination, whereby an index of 0.5-4 suggests no interaction between the antibiotics. 


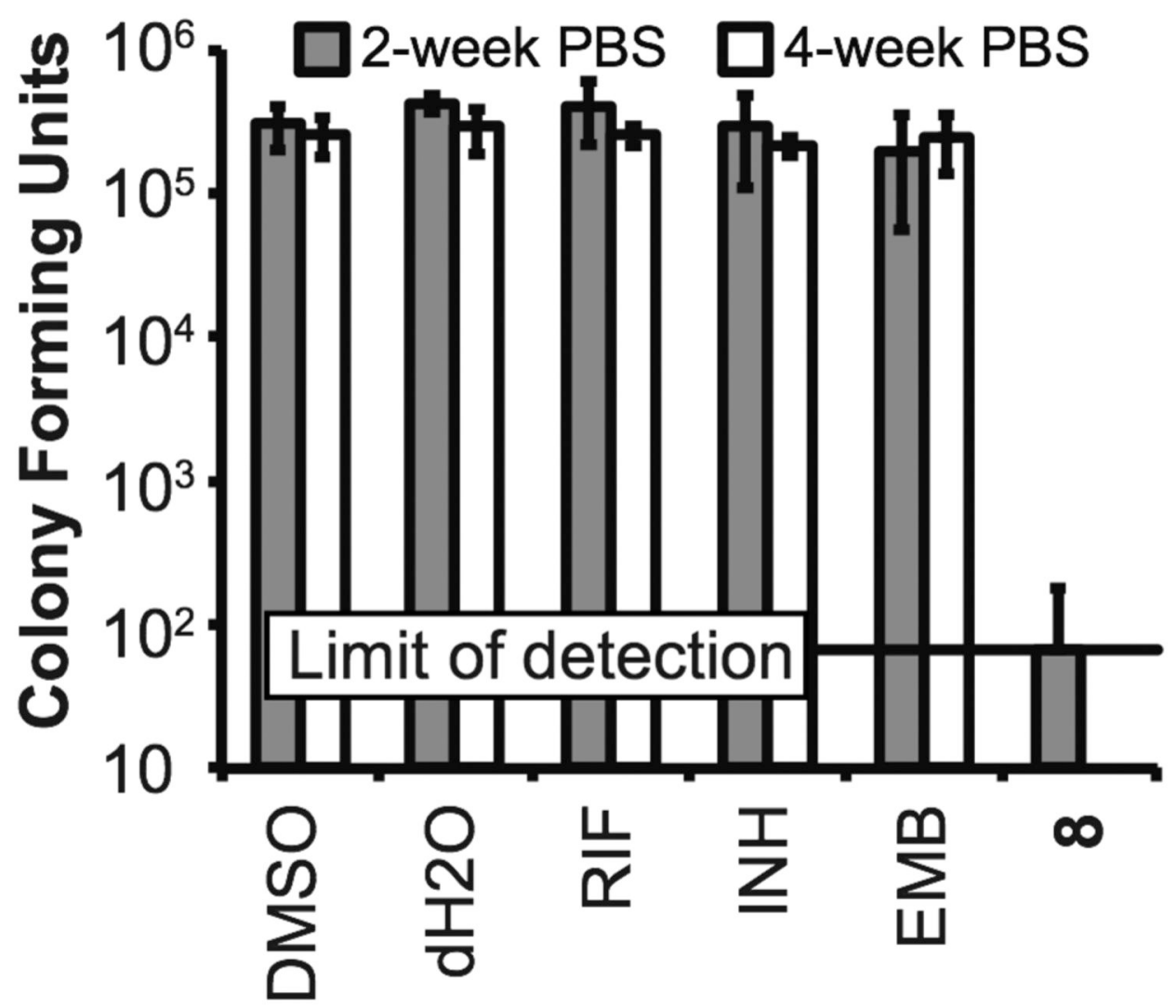

Figure 4 -. Compound 8 is effective against non-growing Mtb.

We treated PBS-starved H37Rv for $5 \mathrm{~d}$ with either solvent vehicle or with RIF, INH, EMB, or 8 at their $10 \times \mathrm{MIC}$ for nutrient-rich grown Mtb. The figure shows the average and standard deviation of three technical replicates each from two independent PBS-starved cultures. 


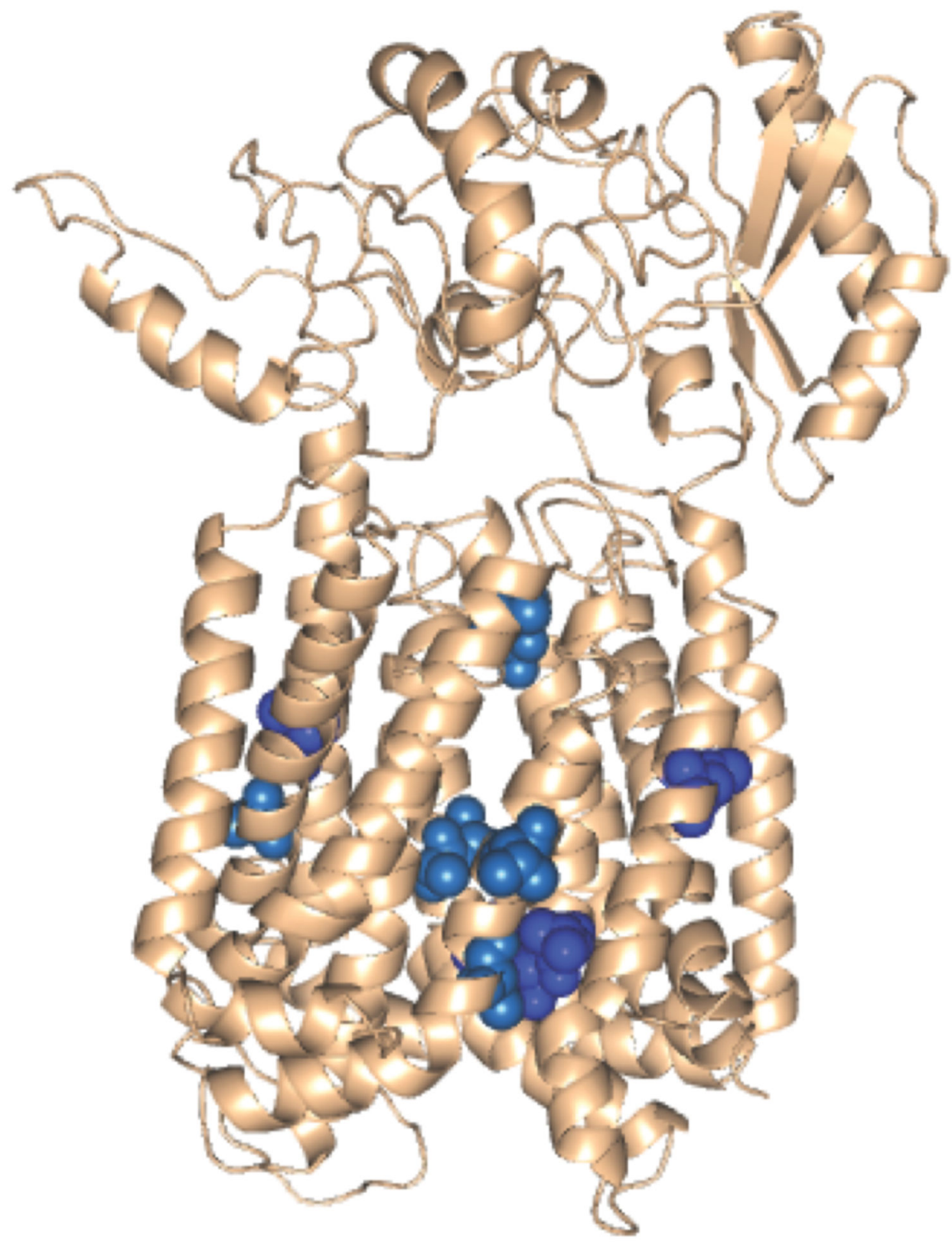

Figure 5 -. In silico model of MmpL3 structure and mapped residues involved in resistance to 8. The core MmpL3 in silico model is shown, including residues 1-728. We generated this model using tools from the Max Planck Bioinformatics toolkit and visualized it with PyMol. ${ }^{27}$ The residues found in the $\mathbf{8}$ screen are dark blue and residues found exclusively in the DA5 screen are light blue. 

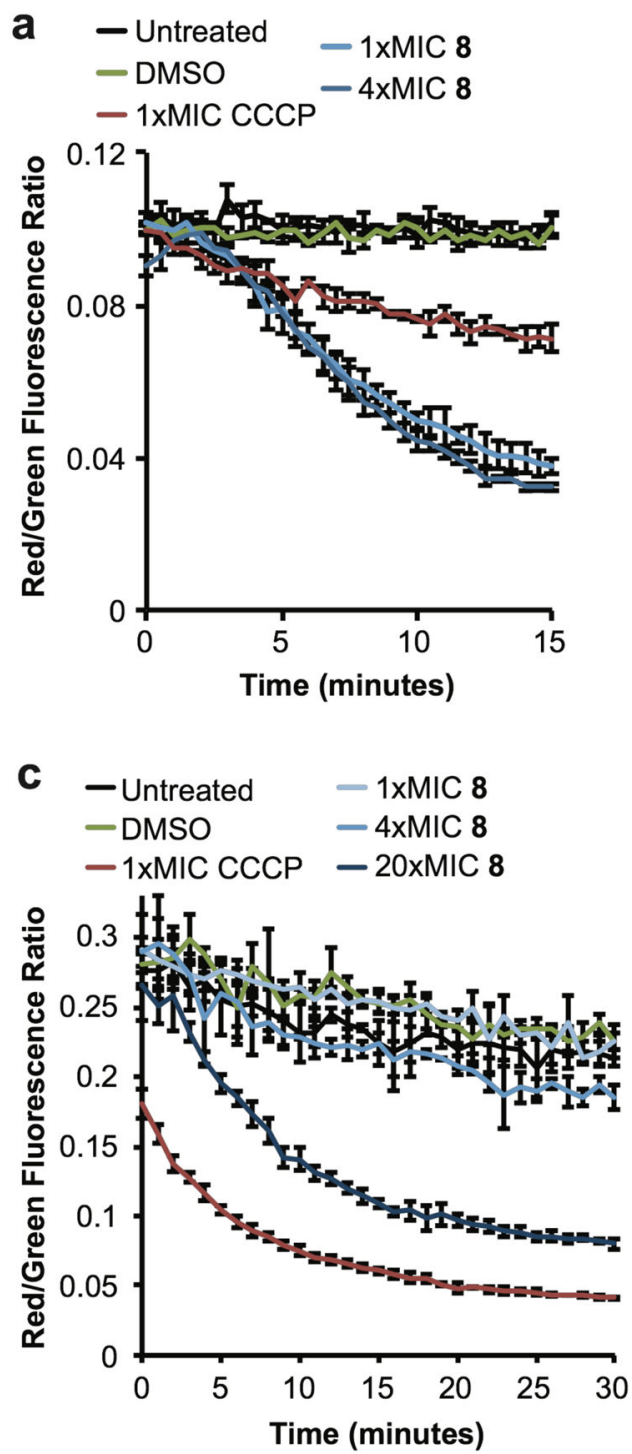
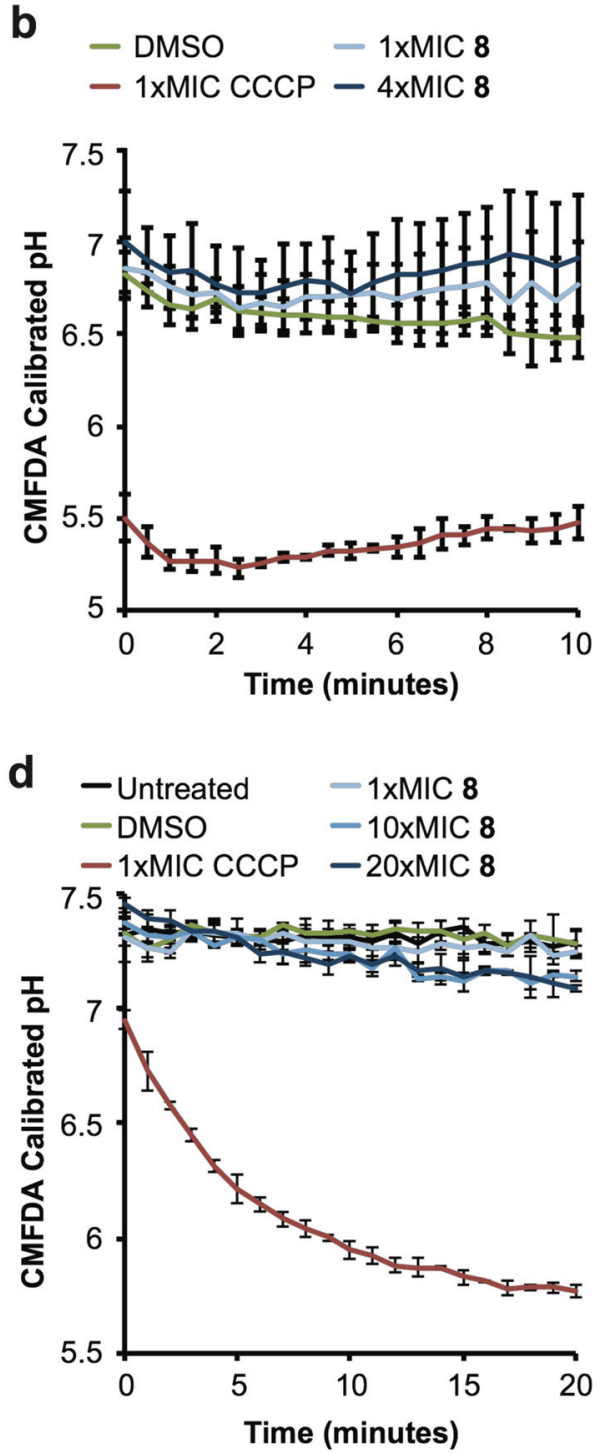

Figure 6 -. Effect of 8 on $\Delta \Psi$ and $\Delta \mathrm{pH}$ in fast- and slow-growing mycobacteria.

We measured $\Delta \Psi(\mathrm{A}, \mathrm{C})$ and the inner bacterial $\mathrm{pH}(\mathrm{B}, \mathrm{D})$ of mycobacteria treated with solvent controls or compounds at the indicated multiples of their in vitro MIC. We measured the $\Delta \Psi$ and $\Delta \mathrm{pH}$ by labeling mycobacteria with $\mathrm{DiOC}_{2}$ and CMFDA, respectively. The (v/v) $\%$ of DMSO was equivalent to the highest amount needed for treatments and kept below $1 \%$ for all experiments. A) In fast-growing M. smegmatis $\mathrm{mc}^{2} 155$, at the $1 \mathrm{xMIC}$ for 8 effectively eliminated $\Delta \Psi$ within 15 min. B) M. smegmatis $\mathrm{mc}^{2}$ treated with 8 does not perturb the $\Delta \mathrm{pH}$ at the concentrations tested against three independent CMFDA labeled cultures. C) In slow-growing $M$. bovis BCG, treatment with the 1xMIC of $\mathbf{8}$ did not rapidly equilibrate $\Delta \Psi$. At 20xMIC, $\Delta \Psi$ depletion occurred similar to the ionophore control CCCP. D) $M$. bovis BCG treated with $\mathbf{8}$ does not rapidly deplete the $\Delta \mathrm{pH}$ at the concentrations tested against three independent CMFDA labeled cultures. 


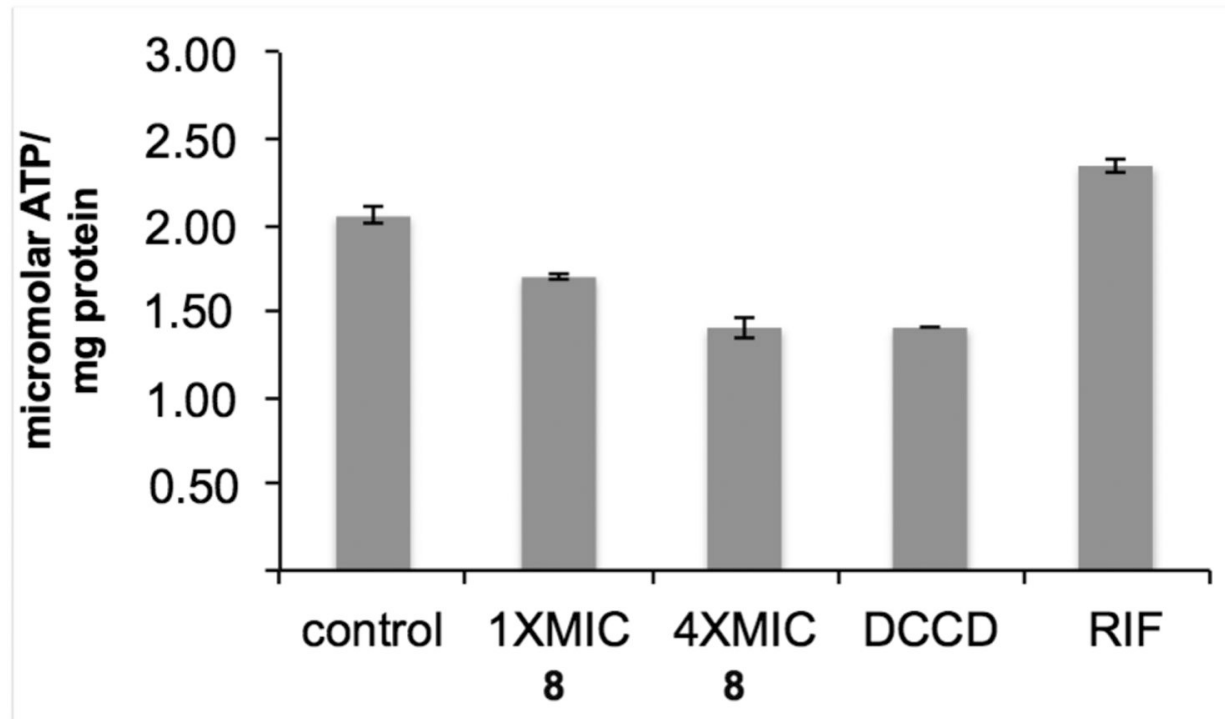

Figure 7 -. Effect of 8 on mycobacterial ATP levels.

M. bovis $\mathrm{BCG}$ was treated overnight with the indicated concentrations of $\mathbf{8}$. The $\mathrm{F}_{0} \mathrm{~F}_{1} \mathrm{ATP}$ synthase inhibitor DCCD was used as a positive control and RIF $(1 \mu \mathrm{M}=65 \mathrm{X} \mathrm{MIC})$ as a negative control. ATP levels are shown normalized to protein concentration. Average and standard deviations of a representative experiment performed in triplicate is shown. 
Table 1 -

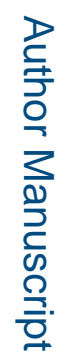

Selective index (SI) of 8 and clinical antibiotics

\begin{tabular}{|l|l|l|l|}
\hline & Rifampicin $(\boldsymbol{\mu g} / \mathbf{m L})$ & Isoniazid $(\boldsymbol{\mu g} / \mathbf{m L})$ & $\mathbf{8}(\boldsymbol{\mu g} / \mathbf{m L})$ \\
\hline IC $_{50}$ THP-1 & 240 & 7000 & 4.4 \\
\hline IC $_{50}$ Vero & 120 & 18000 & 3.9 \\
\hline IC $_{50}$ HFF & ND & ND & 16.1 \\
\hline SI THP-1 & 9600 & 140000 & 3.8 \\
\hline SI Vero & 4800 & 360000 & 3.4 \\
\hline SI HFF & ND & ND & 13.4 \\
\hline
\end{tabular}

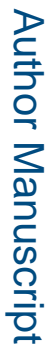

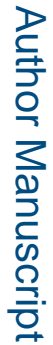

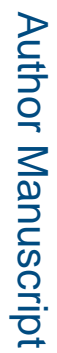


Table 2 -

MICs of standard antibiotics and 8 against drug-resistant Mtb

\begin{tabular}{|l|c|c|c|c|}
\hline $\begin{array}{l}\text { Strain } \\
\text { S - sensitive } \\
\text { R - resistant }\end{array}$ & $\begin{array}{c}\text { RIF } \\
(\mathbf{n g} / \mathbf{m L})\end{array}$ & $\begin{array}{c}\text { INH } \\
(\mathbf{n g} / \mathbf{m L})\end{array}$ & $\begin{array}{c}\mathbf{E M B} \\
(\boldsymbol{\mu g} / \mathbf{m L})\end{array}$ & $\begin{array}{c}\mathbf{8} \\
(\boldsymbol{\mu g} / \mathbf{m L})\end{array}$ \\
\hline T 21 (INH - S) & 12.5 & 50 & 0.5 & 0.58 \\
\hline T 27 (INH - R) & 12.5 & $>800$ & 1.0 & 0.58 \\
\hline Beijing 26 (RIF - S) & 12.5 & 25 & 1.0 & 1.2 \\
\hline Beijing 22 (RIF - R) & $>400$ & 25 & 1.0 & 1.2 \\
\hline Haarlem 23 (RIF - S) & 12.5 & 25 & 1.0 & 1.2 \\
\hline Haarlem 9 (RIF - R) & $>400$ & 25 & 1.0 & 1.2 \\
\hline
\end{tabular}


Table 3 -

Identity of $m m p L 3$ mutations that confer resistance for 8

\begin{tabular}{|l|c|c|c|c|c|}
\hline Resistant Isolates & Base & Codon & $\begin{array}{c}\text { Amino } \\
\text { Acid }\end{array}$ & $\begin{array}{c}\text { DA5 } \\
\mathbf{M I C} \\
(\boldsymbol{\mu g} / \mathbf{m L})\end{array}$ & $\begin{array}{c}\mathbf{8} \\
\mathbf{M I C}(\boldsymbol{\mu g} / \mathbf{m L})\end{array}$ \\
\hline H37Rv & NA & NA & NA & 6.4 & 1.2 \\
\hline TBL-140 \#X3 & 566T & CTG $\rightarrow$ CGG & L189R & 26 & 9.2 \\
\hline DA5 \#4 & 629T & GTG $\rightarrow$ GCG & V210A & 26 & 4.8 \\
\hline DA5 \#6, 8 & 850A & ACG $\rightarrow$ GCG & T284A & 26 & 4.8 \\
\hline DA5 \#11 & 869T & GTG $\rightarrow$ GCG & V290A & 13 & 2.4 \\
\hline TBL-140 \#16 & 1700T & CTG $\rightarrow$ CCG & L567P & 26 & 9.2 \\
\hline DA5 \#10; TBL-140 \#13,14, 17 & 1927G & GTG $\rightarrow$ ATG & V643M & $>26$ & 9.2 \\
\hline DA5 \#12 & 1930T & TTC $\rightarrow$ CTC & F644L & $>26$ & 9.2 \\
\hline TBL-140 \#12 & 1932C & TTC $\rightarrow$ TTG & F644L & $>26$ & 9.2 \\
\hline DA5 \#7 & 2030C & GCG $\rightarrow$ GTG & A677V & 26 & 4.8 \\
\hline DA5 \#9 & 2032G & GCG $\rightarrow$ CCG & A678P & 13 & 2.4 \\
\hline DA5 \#3 & 2095C & CTG $\rightarrow$ ATG & L699M & 26 & 4.8 \\
\hline DA5 \#5 & 2098G & GCC $\rightarrow$ ACC & A700T & $>26$ & 9.2 \\
\hline
\end{tabular}


Table 4 -

Metabolic Stability of 8 versus SQ109 in human and mouse liver microsomes

\begin{tabular}{|l|c|c|c|c|c|c|}
\hline & \multicolumn{3}{|c|}{ human } & \multicolumn{3}{c|}{ mouse } \\
\hline & $\begin{array}{c}\mathbf{t}_{\mathbf{1} / \mathbf{2}} \\
(\mathbf{m i n})\end{array}$ & $\begin{array}{c}\mathbf{C l}_{\text {int }} \\
(\mathbf{m l} / \mathbf{m i n})\end{array}$ & $\begin{array}{c}\text { Predicted } \\
\mathbf{E H}\end{array}$ & $\begin{array}{c}\mathbf{t}_{\mathbf{1} / \mathbf{2}} \\
(\mathbf{m i n})\end{array}$ & $\begin{array}{c}\mathbf{C l}_{\text {int }} \\
(\mathbf{m l} / \mathbf{m i n})\end{array}$ & $\begin{array}{c}\text { Predicted } \\
\mathbf{E H}\end{array}$ \\
\hline $\mathrm{SQ}-109$ & 2.97 & 269 & 0.929 & 2.44 & 1118 & 0.926 \\
\hline $\mathbf{8}$ & 231 & 3.47 & 0.144 & 24.14 & 113 & 0.557 \\
\hline Midazolam & 3.35 & 239 & 0.920 & 2.41 & 1130 & 0.924 \\
\hline
\end{tabular}

